\title{
MRS2016: RIGID MOON ROTATION SERIES IN THE RELATIVISTIC APPROXIMATION
}

\author{
V.V. Pashkevich \\ Central (Pulkovo) Astronomical Observatory of RAS \\ Pulkovskoe shosse, 65/1, 196140, St.Petersburg, Russia \\ e-mail: pashvladvit@yandex.ru
}

\begin{abstract}
The rigid Moon rotation problem is studied for the relativistic (kinematical) case, in which the geodetic perturbations in the Moon rotation are taken into account. As the result of this research the high-precision Moon Rotation Series MRS2016 in the relativistic approximation was constructed for the first time and the discrepancies between the highprecision numerical and the semi-analytical solutions of the rigid Moon rotation were investigated with respect to the fixed ecliptic of epoch J2000, by the numerical and analytical methods. The residuals between the numerical solution and MRS2016 in the perturbing terms of the physical librations do not exceed 80 mas and 10 arc seconds over 2000 and 6000 years, respectively.
\end{abstract}

Keywords: the rigid Moon rotation, ephemerides, the numerical and the semi-analytical solutions, the relativistic approximation.

\section{INTRODUCTION}

In the preceding investigations (Pashkevich and Eroshkin 2011, Pashkevich 2015) the highprecision rigid Moon Rotation Series (designated MRS2011B, MRS2014, respectively) were constructed only for the Newtonian (dynamical) case. In this research the rigid Moon rotation problem is solved for the relativistic (kinematical) case, in which the geodetic perturbations (the most essential relativistic perturbations) in the Moon rotation are taken into account. The main aims of the present research are construction of the high-precision rigid Moon Rotation Series MRS2016A and MRS2016B in the relativistic approximation dynamically adjusted to the JPL DE422/LE422 ephemerides (Folkner 2011), over 2000 and 6000 years, respectively, and investigation of discrepancies between the high-precision numerical and the semianalytical solutions of the rigid Moon rotation problem with respect to the fixed ecliptic of epoch J2000, by the numerical and analytical methods. For these purposes in this study the mathematical model, which were described in our previous investigations is modified (Pashkevich and Eroshkin 2011, Pashkevich 2015) and the iterative algorithm of the problem is applied (Pashkevich 2015).

\section{MATHEMATICAL MODEL}

The problem formularized in the Rodrigues - Hamilton parameters (Pashkevich and Eroshkin 2011), which in this paper are expressed via the perturbing terms of the physical librations of the Moon: 


$$
\begin{array}{ll}
\lambda_{0}=-\cos \frac{\rho+I}{2} \sin \frac{\tau+L_{\mathbb{C}}}{2}, & \lambda_{1}=\sin \frac{\rho+I}{2} \sin \left(\frac{\tau+L_{\mathbb{C}}}{2}-\sigma-\Omega\right), \\
\lambda_{2}=-\sin \frac{\rho+I}{2} \cos \left(\frac{\tau+L_{\mathbb{C}}}{2}-\sigma-\Omega\right), & \lambda_{3}=\cos \frac{\rho+I}{2} \cos \frac{\tau+L_{\mathbb{C}}}{2} .
\end{array}
$$

Here $\tau, \rho$ and $\sigma$ are the perturbing terms of the physical librations of the Moon (Eckhardt 1981) for the fixed ecliptic of epoch J2000 in the longitude, in the inclination and in the node longitude, respectively; $I$ is a constant angle of the inclination of the lunar equator to the fixed ecliptic $\mathrm{J} 2000\left(I \sim 1^{\mathrm{O}} 32^{\prime}\right)$; $L_{\mathbb{C}}$ is the mean longitude of the Moon and $\Omega$ is the mean longitude of the ascending node of its orbit. The Rodrigues-Hamilton parameters determine the orientation of the principal axes of inertia with respect to the Moon-fixed system (the fixes ecliptic and vernal Moon equinox J2000.0).

The differential equations, determining the rigid Moon rotation in the terms of the Rodrigues - Hamilton parameters are:

$$
\begin{aligned}
\left(\begin{array}{c}
\ddot{\lambda}_{0} \\
\ddot{\lambda}_{1} \\
\ddot{\lambda}_{2} \\
\ddot{\lambda}_{3}
\end{array}\right)=\frac{1}{2} K^{-1}\left(\begin{array}{c}
\frac{1}{2} \omega_{R} \\
\dot{\omega}_{R 1} \\
\dot{\omega}_{R 2} \\
\dot{\omega}_{R 3}
\end{array}\right)-\dot{K}\left(\begin{array}{c}
0 \\
\sigma_{X} \\
\sigma_{Y} \\
\sigma_{Z}
\end{array}\right)-\frac{1}{2} K\left(\begin{array}{c}
0 \\
\dot{\sigma}_{X} \\
\dot{\sigma}_{Y} \\
\dot{\sigma}_{Z}
\end{array}\right), K=\left(\begin{array}{ccccc}
-\lambda_{0} & -\lambda_{1} & -\lambda_{2} & -\lambda_{3} \\
-\lambda_{1} & \lambda_{0} & \lambda_{3} & -\lambda_{2} \\
-\lambda_{2} & -\lambda_{3} & \lambda_{0} & \lambda_{1} \\
-\lambda_{3} & \lambda_{2} & -\lambda_{1} & \lambda_{0}
\end{array}\right), \\
K^{-1}=\left(\begin{array}{cccc}
-\lambda_{0} & -\lambda_{1} & -\lambda_{2} & -\lambda_{3} \\
-\lambda_{1} & \lambda_{0} & -\lambda_{3} & \lambda_{2} \\
-\lambda_{2} & \lambda_{3} & \lambda_{0} & -\lambda_{1} \\
-\lambda_{3} & -\lambda_{2} & \lambda_{1} & \lambda_{0}
\end{array}\right), \dot{K}=\left(\begin{array}{cccc}
-\dot{\lambda}_{0} & -\dot{\lambda}_{1} & -\dot{\lambda}_{2} & -\dot{\lambda}_{3} \\
-\dot{\lambda}_{1} & \dot{\lambda}_{0} & \dot{\lambda}_{3} & -\dot{\lambda}_{2} \\
-\dot{\lambda}_{2} & -\dot{\lambda}_{3} & \dot{\lambda}_{0} & \dot{\lambda}_{1} \\
-\dot{\lambda}_{3} & \dot{\lambda}_{2} & -\dot{\lambda}_{1} & \dot{\lambda}_{0}
\end{array}\right) .
\end{aligned}
$$

Here $\omega_{R}^{2}=(\vec{\omega}+\vec{\sigma})^{2} ; \quad \vec{\omega}$ is the Moon Newtonian angular velocity vector; $\vec{\omega}_{R}=\vec{\omega}+\vec{\sigma}$ is the Moon relativistic angular velocity vector; the subscript $R$ corresponds to the relativistic (kinematical) case and the dot denotes a differentiation with respect to time; $\dot{\omega}_{R 1}, \dot{\omega}_{R 2}, \dot{\omega}_{R 3}$ are the first derivatives of the angular velocity projections of the Moon relativistic angular velocity vector $\vec{\omega}_{R}=\vec{\omega}+\vec{\sigma}$ on the Moon principal axes of inertia; $\sigma_{X}, \sigma_{Y}, \sigma_{Z}$ are the projections of the vector $\vec{\sigma}$ on the axes of the non-rotating ecliptic reference system; $\vec{\sigma}$ is the vector of the angular velocity of the Moon geodetic rotation, which is defined by the following expression (Eroshkin and Pashkevich 2009):

$$
\vec{\sigma}=\left.\sum_{j \neq \mathbb{\complement}} \frac{G m_{j}}{c^{2} \mid \vec{R}_{j}-\vec{R} \rrbracket}\right|^{3}\left\{\left(2 \dot{\vec{R}}_{j}-\frac{3}{2} \dot{\vec{R}}_{\mathbb{}}\right) \times\left(\vec{R}_{j}-\vec{R}_{\S}\right)\right\} .
$$

Here $c$ is the velocity of light; $G$ is the gravitational constant; $m_{j}$ is the mass of the $j$-th disturbing body (the Sun, the Earth and the major planets); $\vec{R}_{j}, \dot{\vec{R}}_{j}, \vec{R}_{\mathbb{}}, \dot{\vec{R}}_{\mathbb{C}}$ are the vectors of the barycentric position and velocity of bodies $j$ and the Moon, respectively. The symbol $\times$ means a vector product; the subscript \& corresponds to the Moon; the subscript $j$ corresponds to the Earth, the major planets and the Sun.

The first derivatives of the angular velocity components are given by the following equations: 


$$
\begin{aligned}
& \dot{\omega}_{R 1}=-\alpha \omega_{R 2} \omega_{R 3}+\frac{1}{A} \sum_{j \neq \mathbb{\complement}}\left(z_{R j} \frac{\partial U_{j}}{\partial y_{R j}}-y_{R j} \frac{\partial U_{j}}{\partial z_{R j}}\right), \\
& \dot{\omega}_{R 2}=\beta \omega_{R 1} \omega_{R 3}+\frac{1}{B} \sum_{j \neq \mathbb{C}}\left(x_{R j} \frac{\partial U_{j}}{\partial z_{R j}}-z_{R j} \frac{\partial U_{j}}{\partial x_{R j}}\right), \\
& \dot{\omega}_{R 3}=-\gamma \omega_{R 1} \omega_{R 2}+\frac{1}{C} \sum_{j \neq \mathbb{C}}\left(y_{R j} \frac{\partial U_{j}}{\partial x_{R j}}-x_{R j} \frac{\partial U_{j}}{\partial y_{R j}}\right) .
\end{aligned}
$$

Here $U_{j}$ is the force function of the gravitational interaction of the Moon with the $j$-th disturbing body (the Earth, the Sun and the major planets) (Pashkevich and Eroshkin 2011); the values of the Moon principal moments of inertia $A, B, C$ and $\alpha=(C-B) / A=(\beta-\gamma) /(1-\beta \gamma), \beta=(C-A) / B=631.26 \cdot 10^{-6}, \gamma=(B-A) / C=227.37 \cdot 10^{-6}$ were taken from (Eckhardt 1981); $x_{R j}, y_{R j}, z_{R j}$ are the projections of the lunarcentric vector $\vec{r}_{R j}$ of the $j$-th disturbing body into axes of the Moon-fixed reference frame; $\omega_{R 1}, \omega_{R 2}, \omega_{R 3}$ are the projections of the Moon relativistic angular velocity vector $\vec{\omega}_{R}=\vec{\omega}+\vec{\sigma}$ on the Moon principal axes of inertia. The orbital motions of the disturbing bodies are defined by the JPL DE422/LE422 ephemerides.

The vector-matrix equation expresses the relations between the components of the angular velocity vector and the Rodrigues - Hamilton parameters:

$$
\left(\begin{array}{c}
0 \\
\omega_{R 1} \\
\omega_{R 2} \\
\omega_{R 3}
\end{array}\right)=\left(\begin{array}{c}
0 \\
\omega_{1}+\sigma_{1} \\
\omega_{2}+\sigma_{2} \\
\omega_{3}+\sigma_{3}
\end{array}\right)=2 K\left(\left(\begin{array}{c}
\dot{\lambda}_{0} \\
\dot{\lambda}_{1} \\
\dot{\lambda}_{2} \\
\dot{\lambda}_{3}
\end{array}\right)+\frac{1}{2} K\left(\begin{array}{c}
0 \\
\sigma_{X} \\
\sigma_{Y} \\
\sigma_{Z}
\end{array}\right)\right) .
$$

Here $\omega_{1}, \omega_{2}, \omega_{3}$ are the projections of the Moon Newtonian angular velocity vector $\vec{\omega}$ on the Moon principal axes of inertia; $\sigma_{1}, \sigma_{2}, \sigma_{3}$ are the components of the vector $\vec{\sigma}$ in the Moonfixed system.

The transformation of the DE422/LE422 ephemerides vector $\quad \vec{r}_{j}^{422}\left(x_{j}^{422}, y_{j}^{422}, z_{j}^{422}\right)$ to the vector $\vec{r}_{R j}\left(x_{R j}, y_{R j}, z_{R j}\right)$ in the Moon-fixed system is performed in two steps:

\section{1-st step:}

The transformation of the DE422/LE422 ephemerides vector $\vec{r}_{j}^{422}\left(x_{j}^{422}, y_{j}^{422}, z_{j}^{422}\right)$ to the vector $\vec{r}_{j}\left(x_{j}, y_{j}, z_{j}\right)$ in the Moon-fixed system, when this problem is solved in the Newtonian approximation:

$$
\begin{aligned}
\left(\begin{array}{l}
x_{j} \\
y_{j} \\
z_{j}
\end{array}\right) & =M p\left(\varepsilon_{0}\right) r(\Delta)\left(\begin{array}{l}
x_{j}^{422} \\
y_{j}^{422} \\
z_{j}^{422}
\end{array}\right) \text {, where } \Delta=-0 " .05294, \varepsilon_{0}=23^{\circ} 26^{\prime} 21^{\prime \prime} .40928, \\
r(a) & =\left(\begin{array}{ccc}
\cos a & \sin a & 0 \\
-\sin a & \cos a & 0 \\
0 & 0 & 1
\end{array}\right), p(a)=\left(\begin{array}{ccc}
1 & 0 & 0 \\
0 & \cos a & \sin a \\
0 & -\sin a & \cos a
\end{array}\right), M=[K K]_{11}
\end{aligned}
$$


Algebraic addendum of the element $k k_{11}$ matrix product $K K$ is the following:

$$
[K K]_{11}=\left(\begin{array}{ccc}
\lambda_{0}^{2}+\lambda_{1}^{2}-\lambda_{2}^{2}-\lambda_{3}^{2} & 2\left(\lambda_{0} \lambda_{3}+\lambda_{1} \lambda_{2}\right) & 2\left(\lambda_{1} \lambda_{3}-\lambda_{0} \lambda_{2}\right) \\
2\left(\lambda_{1} \lambda_{2}-\lambda_{0} \lambda_{3}\right) & \lambda_{0}^{2}-\lambda_{1}^{2}+\lambda_{2}^{2}-\lambda_{3}^{2} & 2\left(\lambda_{0} \lambda_{1}+\lambda_{2} \lambda_{3}\right) \\
2\left(\lambda_{0} \lambda_{2}+\lambda_{1} \lambda_{3}\right) & 2\left(\lambda_{2} \lambda_{3}-\lambda_{0} \lambda_{1}\right) & \lambda_{0}^{2}-\lambda_{1}^{2}-\lambda_{2}^{2}+\lambda_{3}^{2}
\end{array}\right) .
$$

Matrix product $K K$ is the following:

$$
K K=\left(\begin{array}{cccc}
1 & 0 & 0 & 0 \\
0 & \lambda_{0}^{2}+\lambda_{1}^{2}-\lambda_{2}^{2}-\lambda_{3}^{2} & 2\left(\lambda_{0} \lambda_{3}+\lambda_{1} \lambda_{2}\right) & 2\left(\lambda_{1} \lambda_{3}-\lambda_{0} \lambda_{2}\right) \\
0 & 2\left(\lambda_{1} \lambda_{2}-\lambda_{0} \lambda_{3}\right) & \lambda_{0}^{2}-\lambda_{1}^{2}+\lambda_{2}^{2}-\lambda_{3}^{2} & 2\left(\lambda_{0} \lambda_{1}+\lambda_{2} \lambda_{3}\right) \\
0 & 2\left(\lambda_{0} \lambda_{2}+\lambda_{1} \lambda_{3}\right) & 2\left(\lambda_{2} \lambda_{3}-\lambda_{0} \lambda_{1}\right) & \lambda_{0}^{2}-\lambda_{1}^{2}-\lambda_{2}^{2}+\lambda_{3}^{2}
\end{array}\right) .
$$

\section{2-nd step:}

The transformation of the Newtonian vector $\vec{r}_{j}\left(x_{j}, y_{j}, z_{j}\right)$ to the relativistic vector $\vec{r}_{R j}\left(x_{R j}, y_{R j}, z_{R j}\right)$ by means of the geodetic rotation, when this problem is solved in the relativistic (kinematical) case, is obtained in the result of integration of the relation between the time changes of the vector $\vec{r}_{j}$ with respect to two coordinate systems, one of which rotates relatively to another with angular velocity $\vec{\sigma}$ :

$$
\frac{d \vec{r}_{j}^{*}}{d t}=\frac{d \vec{r}_{j}}{d t}-\vec{\sigma} \times \vec{r}_{j}
$$

over the time interval $\left[t_{1969}, t\right]$. As a result of integration of eq. (11), the following expression is obtained:

$$
\vec{r}_{j}^{*}=\vec{r}_{j}-\int_{t_{1969}}^{t} \vec{\sigma} \times \vec{r}_{j} d t,
$$

where $t_{1969}=\operatorname{JD} 2440400.5\left(1969,28\right.$ June, $0^{\mathrm{h}}$ ET) is the initial epoch of the rigid Moon Rotation Series. Since this transformation is a rotational transformation, then $\left|\vec{r}_{j}^{*}\right|=\left|\vec{r}_{j}\right|$.

The iterative algorithm of the problem solution is described in detail in the paper (Pashkevich 2015). The initial conditions of the numerical integration for first iteration of the iterative algorithm are calculated from the composite semi-analytical theory of the rigid Moon rotation MRS2016, which include the semi-analytical solution of the rigid Moon rotation (MRS2011B for 2000 years or MRS2014 for 6000 years) and the most essential terms (Table 1) of the Moon geodetic rotation (Pashkevich 2016). MRS2016 $6_{0}$ is used also for the comparison with numerical solution of first iteration of the iterative algorithm. 
Table 1. The most essential terms of the Moon geodetic rotation in the perturbing terms of the physical librations (Pashkevich 2016).

\begin{tabular}{|c|c|c|}
\hline \multicolumn{2}{|r|}{ The secular terms } & The periodic terms \\
\hline & $\Delta \tau(\mu \mathrm{as})$ & $\Delta \tau(\mu$ as) \\
\hline $\mathrm{T}$ & 19494124.5437 & $(-34.279-7.559 \mathrm{~T}+\ldots) \sin \lambda_{3}+(-149.201+5.683 \mathrm{~T}+\ldots) \cos \lambda_{3}+$ \\
\hline $\mathrm{T}^{2}$ & 12.3515 & $(30.212-0.001 \mathrm{~T}+\ldots) \sin D+(0.001+0.001 \mathrm{~T}+\ldots) \cos D+\ldots$ \\
\hline $\mathrm{T}^{3}$ & -565.0947 & \\
\hline & $\Delta \rho(\mu \mathrm{as})$ & $\Delta \rho(\mu \mathrm{as})$ \\
\hline $\mathrm{T}$ & 300.5067 & $\left(-9 \cdot 10^{-4}-0.008 \mathrm{~T}+\ldots\right) \sin \lambda_{3}+\left(-3 \cdot 10^{-4}-0.025 \mathrm{~T}+\ldots\right) \cos \lambda_{3}+$ \\
\hline $\mathrm{T}^{2}$ & 1780.4437 & $(-0.004+0.010 \mathrm{~T}+\ldots) \sin D+(0.005+0.007 \mathrm{~T}+\ldots) \cos D+\ldots$ \\
\hline $\mathrm{T}^{3}$ & 3126.0421 & \\
\hline & $\Delta(\mathrm{I} \sigma)(\mu \mathrm{as})$ & $\Delta(\mathrm{I} \sigma)(\mu \mathrm{as})$ \\
\hline $\mathrm{T}$ & -6544.4452 & $(0.013-0.111 \mathrm{~T}+\ldots) \sin \lambda_{3}+(0.052-0.496 \mathrm{~T}+\ldots) \cos \lambda_{3}+$ \\
\hline $\mathrm{T}^{2}$ & 36212.8892 & $(-0.016+0.093 \mathrm{~T}+\ldots) \sin D+(-0.006+0.004 \mathrm{~T}+\ldots) \cos D+\ldots$ \\
\hline $\mathrm{T}^{3}$ & -27286.6251 & \\
\hline
\end{tabular}

In Table 1: $D=\lambda_{10}-\lambda_{3}+180^{\circ}, \lambda_{3}$ is the mean longitude of the Earth; $\lambda_{10}$ is the mean geocentric longitude of the Moon; $\mathrm{T}$ is the mean Dynamical Barycentric Time (TDB) measured in thousands Julian years (tjy) (of 365250 days) from J2000. The mean longitudes of the Earth and the Moon were taken from (Brumberg and Bretagnon 2000).

\section{RESULTS}

A. The investigation of the rigid Moon rotation over 2000 years time interval.

The discrepancies of the comparison between the numerical solutions and semi-analytical series MRS2011B for Newtonian (dynamical) case in the previous investigation (Pashkevich and Eroshkin 2011) are depicted in Figure 1.

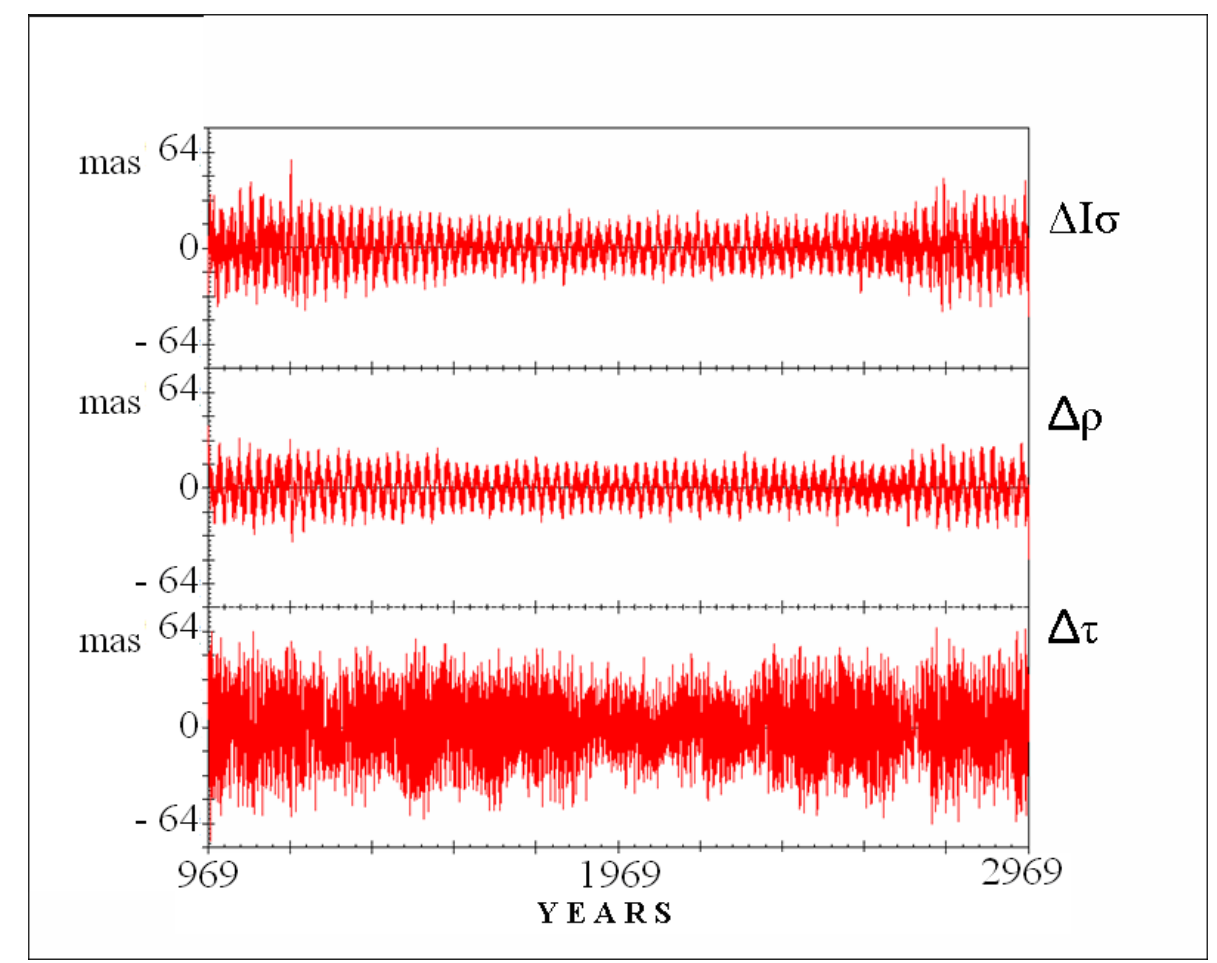

Fig. 1. Numerical solution minus MRS2011B solution (for Newtonian (dynamical) case) 
The behaviour of the discrepancies between the new numerical solution and new semianalytical solution MRS2016A for the relativistic (kinematical) case in this investigation are depicted in Figure 2. These discrepancies (presented in Figure $1-2$ ) are very similar and do not exceed 80 mas over 2000 years for $\Delta \tau, 40$ mas over 2000 years for $\Delta \mathrm{I} \sigma$ and 20 mas over 2000 years for $\Delta \rho$.

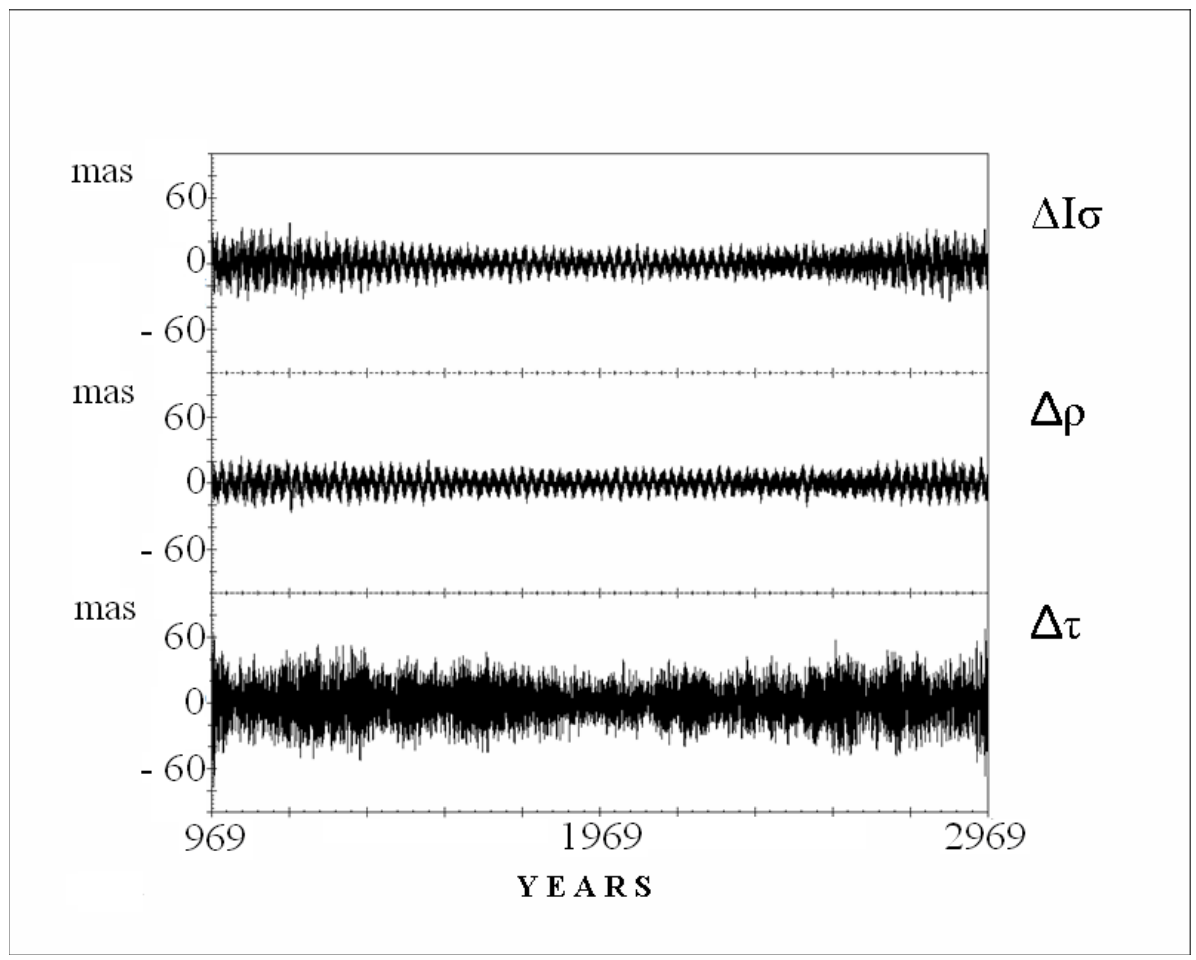

Fig. 2. Numerical solution minus MRS2016A solution (for the relativistic (kinematical) case)

$B$. The investigation of the rigid Moon rotation over 6000 years time interval.

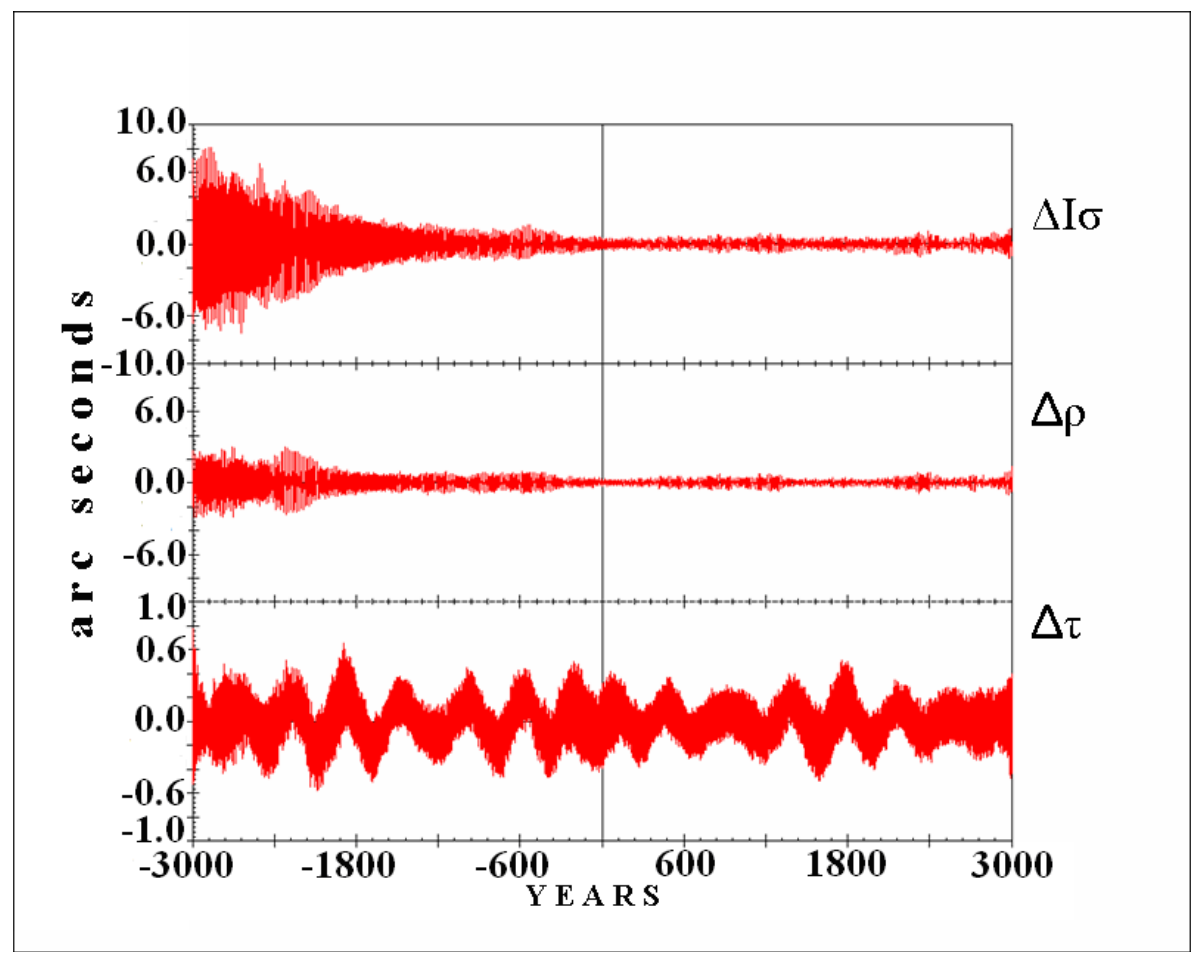

Fig. 3. Numerical solution minus MRS2014 solution (for Newtonian (dynamical) case) 
The discrepancies of the comparison between the numerical solutions and semi-analytical series MRS2014 for Newtonian (dynamical) case in the previous investigation (Pashkevich 2015) are depicted in Figure 3.

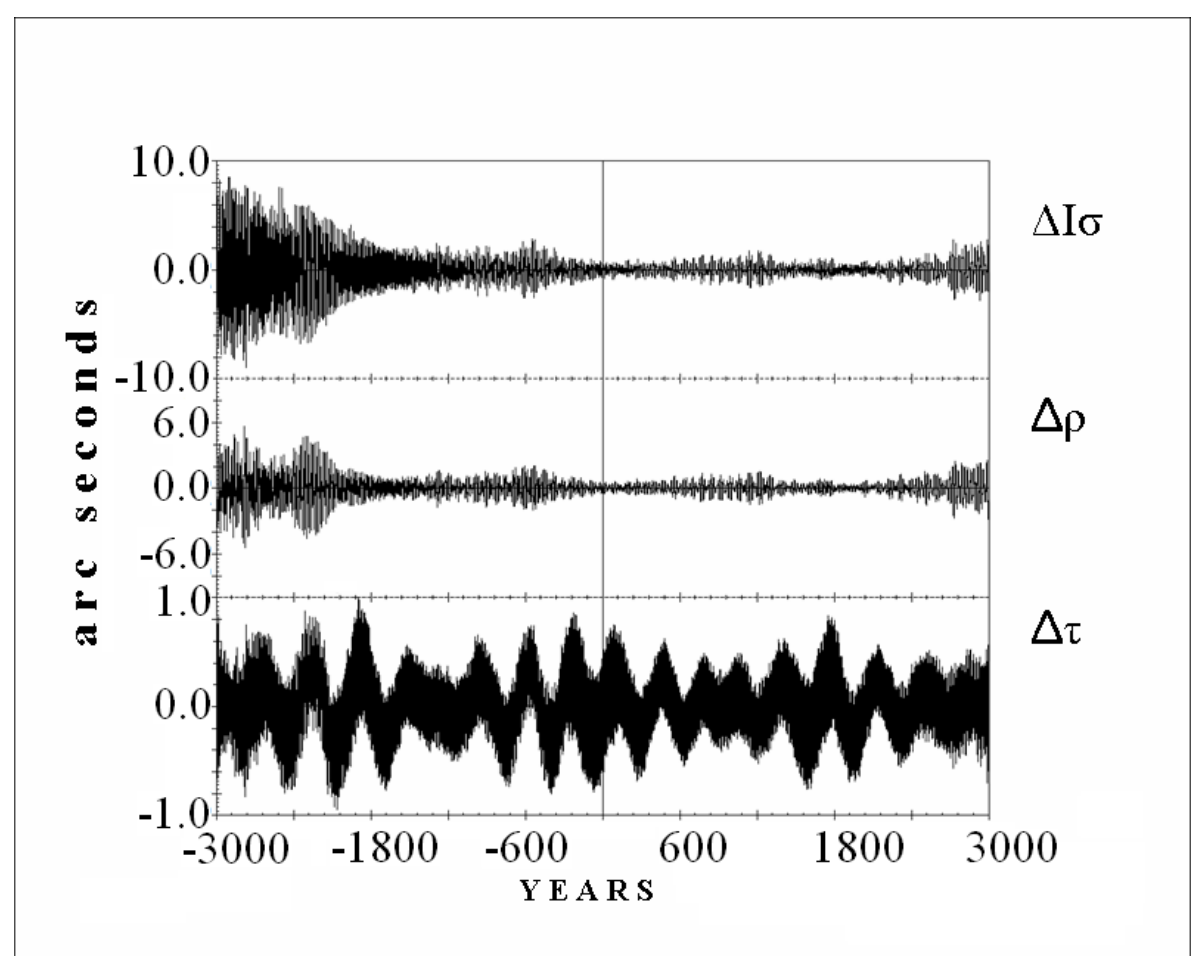

Fig. 4. Numerical solution minus MRS2016B solution (for the relativistic (kinematical) case)

The behaviour of the discrepancies between the new numerical solution and new semianalytical solution MRS2016B for the relativistic (kinematical) case in this investigation are depicted in Figures 4. These discrepancies do not exceed 8 arc seconds over 6000 years for $\Delta \mathrm{I} \sigma, 4$ arc seconds over 6000 years for $\Delta \rho$ and 1 arc second over 6000 years for $\Delta \tau$ (Figures 3 $-4)$.

\section{CONCLUSIONS}

The new high-precision rigid Moon Rotation Series MRS2016A and MRS2016B in the relativistic approximation dynamically adjusted to the JPL DE422/LE422 ephemerides over 2000 and 6000 years have been constructed, respectively for the first time.

MRS2016 include about 1520 periodical terms. Discrepancies between the numerical solution and MRS2016 do not exceed 80 mas over 2000 and 10 arc seconds over 6000 years.

Acknowledgements. The investigation was carried out at the Central (Pulkovo) Astronomical Observatory of the Russian Academy of Science and the Space Research Centre of the Polish Academy of Science, under a financial support of the cooperation between the Polish and Russian Academies of Sciences, Theme No 34 and Aleksander Brzeziński personal grant the Polish national science foundation NCN under grant No. DEC-2012/05/B/ST10/02132. 


\section{REFERENCES}

Eckhardt D.H. (1981) Theory of Libration of the Moon, The Moon and the planets, 25, pp.3-49.

Brumberg V.A., Bretagnon P. (2000) Kinematical Relativistic Corrections for Earth's Rotation Parameters, in Proc. of IAU Colloquium 180, eds. K.Johnston, D. McCarthy, B. Luzum and G. Kaplan, U.S. Naval Observatory, pp. 293-302.

Pashkevich V.V., Eroshkin G.I. (2005) Application of the spectral analysis for the mathematical modelling of the rigid Earth rotation, Artificial Satellites, Warszawa, 2005, Vol. 40, No 4, pp. 251-259.

Eroshkin G.I., Pashkevich V.V. (2009) On the geodetic rotation of the major planets, the Moon and the Sun, Artificial Satellites, Warszawa, 2009, Vol. 44, No. 2, pp. 43-52.

Pashkevich V.V., Eroshkin G.I. (2010) Application of the spectral analysis for modeling the rotations of the Moon, Artificial Satellites, Warszawa, 2010, Vol. 45, No 4, pp.153-162.

Folkner W.F. (2011) JPL Planetary and Lunar Ephemerides: Export Information http://iaucomm4. jpl.nasa.gov/README.html.

Pashkevich V.V., Eroshkin G.I. (2011) Construction of the new high-precision Moon rotation series at a long time intervals, Artificial Satellites, Warszawa, 2011, Vol. 46. No. 2. (DOI: 10.2478/v10018-011-0013-3), pp. 63-73.

Pashkevich V.V. (2015) RERS2014 and MRS2014: new high-precision rigid Earth rotation and Moon rotation series, Artificial Satellites, Warszawa, 2015, Vol. 50, No. 1 (DOI: 10.1515/arsa-2015-0003), pp. 35-40.

Pashkevich V.V. (2016) New high-precision values of the geodetic rotation of the major planets, Pluto, the Moon and the Sun, Artificial Satellites, Warszawa, 2016, Vol. 51, No. 2 (DOI: 10.1515/arsa-2016-0006), pp. 61-73.

Received: 2016-10-26,

Reviewed: 2017-01-27 and 2017-02-17,

Accepted: 2017-02-24. 